\title{
Prevalence and risk factors of gammaherpesvirus infection in domestic cats in Central Europe
}

\author{
Reinhard Ertl ${ }^{1 *}$, Melanie Korb ${ }^{1}$, Ines Langbein-Detsch ${ }^{2}$ and Dieter Klein ${ }^{1}$
}

\begin{abstract}
Background: Gammaherpesviruses (GHVs) are a large group of dsDNA viruses that can infect humans and several animal species. The two human GHVs, Epstein-Barr virus and Kaposi's sarcoma-associated herpesvirus are known for their oncogenic properties in individuals with immunodeficiency. Recently, the first feline GHV, Felis catus gammaherpesvirus 1 (FcaGHV1) was discovered and frequently found in domestic cats in Australia, Singapore and the USA. FcaGHV1 is more likely to be detected in cats co-infected with the feline immunodeficiency virus (FIV).

Findings: The prevalence of FcaGHV1 in pet cats from Germany and Austria was $16.2 \%$ ( $95 \% \mathrm{Cl}=12.38-20.02)$. The odds for $G H V$ infection were greater for FIV positive $(O R=4.5)$, male $(O R=13.32)$ and older $(O R=2.36)$ cats. Furthermore, FcaGHV1 viral loads were significantly higher in FIV-infected cats compared to matched controls.

Conclusions: GHV infections are common in domestic cats in Central Europe. The worldwide distribution of FcaGHV1 can be assumed. A potential role as a co-factor in FIV-induced pathogeneses is supported.
\end{abstract}

\section{Background}

Gammaherpesviruses (GHVs) are a large and widely distributed group of double-stranded DNA viruses within the Herpesviridae family [1]. The two human GHVs, Epstein-Barr virus (EBV) and Kaposi's sarcoma-associated herpesvirus (KSHV) are of particular importance due to their association with the development of cancer in immunosuppressed HIV patients [2]. In 2014, the first GHVs native to feline species (domestic cats, bobcats and pumas) were discovered in the USA [3]. Felis catus gammaherpesvirus 1 (FcaGHV1) was then frequently detected in domestic cats in Australia, Singapore and the USA [3, 4]. Interestingly, the prevalence of FcaGHV1 is higher in cats co-infected with the feline immunodeficiency virus (FIV) [4]. This feline retrovirus is a widespread pathogen in cats causing an AIDS-like immunodeficiency syndrome [5]. Furthermore, FIV-infected animals have a higher risk for the development of lymphoid malignancies, especially lymphomas [6]. However, the underlying mechanisms of FIV associated malignancies are not completely understood.

\footnotetext{
* Correspondence: reinhard.ertl@vetmeduni.ac.at

${ }^{1}$ VetCore Facility for Research, University of Veterinary Medicine Vienna, Vienna, Austria

Full list of author information is available at the end of the article
}

Thus, similar to the human GHVs, FcaGHV1 in cats might be a co-factor for malignant transformation in immunocompromised individuals [7]. In this report, we assess the prevalence of FcaGHV1 among client-owned pet cats in Germany and Austria and evaluate potential risk factors such as FIV co-infection.

\section{Results}

A total of 462 cats from Germany $(n=402)$ and Austria $(n=60)$ were tested for the presence of FcaGHV1 DNA by quantitative PCR (qPCR) and FIV provirus DNA (qPCR and conventional PCR). The prevalence of FcaGHV1 was $16.2 \%(95 \% \mathrm{CI}=12.38-20.02)$ in FIV negative cats from both countries $(\mathrm{n}=358)$. Among FIV-infected cats (all from Germany, $\mathrm{n}=104$ ) a coinfection with FcaGHV1 could be detected in $40.4 \%$ (95\% CI $=30.95-49.81)$ of the animals. All tested Austrian cats were FIV negative and therefore excluded from the risk factor analysis. Odds ratios (OR) were calculated to evaluate risk factors for FcaGHV1 infection. FIV-infected cats in Germany showed a significantly higher probability for being FcaGHV1 positive $(\mathrm{OR}=4.5)$ compared to non-infected animals (Table 1). Other factors that could be associated with FcaGHV1 infection were being 
Table 1 Prevalence and risk factors of FcaGHV1 infection in domestic cats in Germany and Austria; based on the results of chi square tests and the calculation of odds ratios

\begin{tabular}{|c|c|c|c|c|c|c|}
\hline Factor & Category & Prevalence FcaGHV1 & $P$ value & Odds ratio & $95 \% \mathrm{Cl}$ & Origin \\
\hline \multirow[t]{2}{*}{ FIV status } & Negative & 39/298 (13.1 \%) & Ref & - & - & Germany \\
\hline & Positive & 42/104 (40.4 \%) & $<0.0001^{*}$ & 4.499 & $2.684-7.541$ & Germany \\
\hline \multirow[t]{2}{*}{ Sex } & Female & 10/123 (8.1 \%) & Ref & - & - & Germany \\
\hline & Male & 99/183 (54.1\%) & $<0.0001^{*}$ & 13.320 & $6.553-27.07$ & Germany \\
\hline \multirow[t]{2}{*}{ Age } & $\leq 5$ years & 19/152 (12.5\%) & Ref & - & - & Germany \\
\hline & $>5.5$ years & $31 / 123$ (25.2 \%) & $0.0066^{*}$ & 2.359 & $1.256-4.429$ & Germany \\
\hline \multirow[t]{4}{*}{ Neuter status } & Male intact & 17/63 (27 \%) & Ref & - & - & Germany \\
\hline & Male neutered & $36 / 120$ (30 \%) & 0.6691 & 1.160 & $0.588-2.289$ & Germany \\
\hline & Female intact & $3 / 55(5.5 \%)$ & Ref & - & - & Germany \\
\hline & Female neutered & 7/68 (10.3 \%) & 0.3288 & 1.989 & $0.489-8.087$ & Germany \\
\hline \multirow[t]{2}{*}{ Health status } & Healthy' & 8/31 (25.8 \%) & Ref & - & - & Austria \\
\hline & Chronically ill ${ }^{2}$ & $11 / 29(37.9 \%)$ & 0.3130 & 1.757 & $0.585-5.279$ & Austria \\
\hline
\end{tabular}

$\mathrm{Cl}=$ confidence interval, Ref $=$ Reference category, ${ }^{*}=$ statistically significant $(\mathrm{p}<0.05),{ }^{1}$ Includes cats examined for injury, intoxication or routine checkup, ${ }^{2} \mathrm{Cats}$ diagnosed with chronic condition: cardiovascular disease $(n=6)$, tumor $(n=14)$, chronic kidney disease $(n=5)$, chronic inflammation $(n=3)$, neurological disorder $(n=1)$. Only animals with the relative information are enlisted

male $(\mathrm{OR}=13.32)$ and an increasing age $(\mathrm{OR}=2.36)$. No association could be detected for the neuter status of the cats. Detailed clinical data were only available for the cats from Austria. These were grouped according to their health status (healthy vs. chronically ill). However, no significant differences could be found between the two groups. The numbers of FcaGHV1 and FIV DNA copies in the cat blood were measured by qPCR and analyzed for the individual groups. The FcaGHV1 viral loads were significantly $(\mathrm{p}=0.0018)$ higher in FIV-infected cats (median FcaGHV1 viral load: 529 copies $/ 10^{6}$ cells) compared to non-infected cats (221 copies $/ 10^{6}$ cells; Fig. 1). Both groups have been matched for age and sex. FcaGHV1 copy numbers, however, did not correlate with the numbers of FIV copies in the coinfected animals (Spearman $r=-0.024$; Additional file 1). Parts of the FcaGHV1 glycoprotein B (gB) gene were sequenced from 12 German and 6 Austrian virus isolates. The gB sequences of all German and 5 Austrian samples (GenBank: KP862648, KP862649) were identical to those of previously reported strains from Australia and the USA $[3,4]$. In one Austrian isolate (KT241042), a single, synonymous nucleotide polymorphism (adenine to guanine) was detected at nucleotide position 249 of the partial US gB sequence (KF840715) published by Troyer et al. [3].

\section{Discussion}

All samples analyzed in this study have been taken from pet cats during a veterinary medical examination. Clinical data were only available for a part of the animals. On account of this, only prevalence rates and already known risk factors for FcaGHV1 infection were evaluated [4]. The prevalence of FcaGHV1 among FIV negative cats in Germany and Austria (16.2\%) is similar to previously reported data from Australia (11.4\%), Singapore (9.6 \%) and the USA (19.1\%) [4]. In comparison, FIV-infected animals from Germany were 4 times more likely to be tested FcaGHV1 positive. Furthermore, virus DNA loads were significantly higher in the FIV positive group. These results are congruent with previous reports [4]. Taken together, these findings indicate possible pathogenic interactions between FcaGHV1 and FIV, similar to the known associations of the human herpesviruses EBV and KSHV

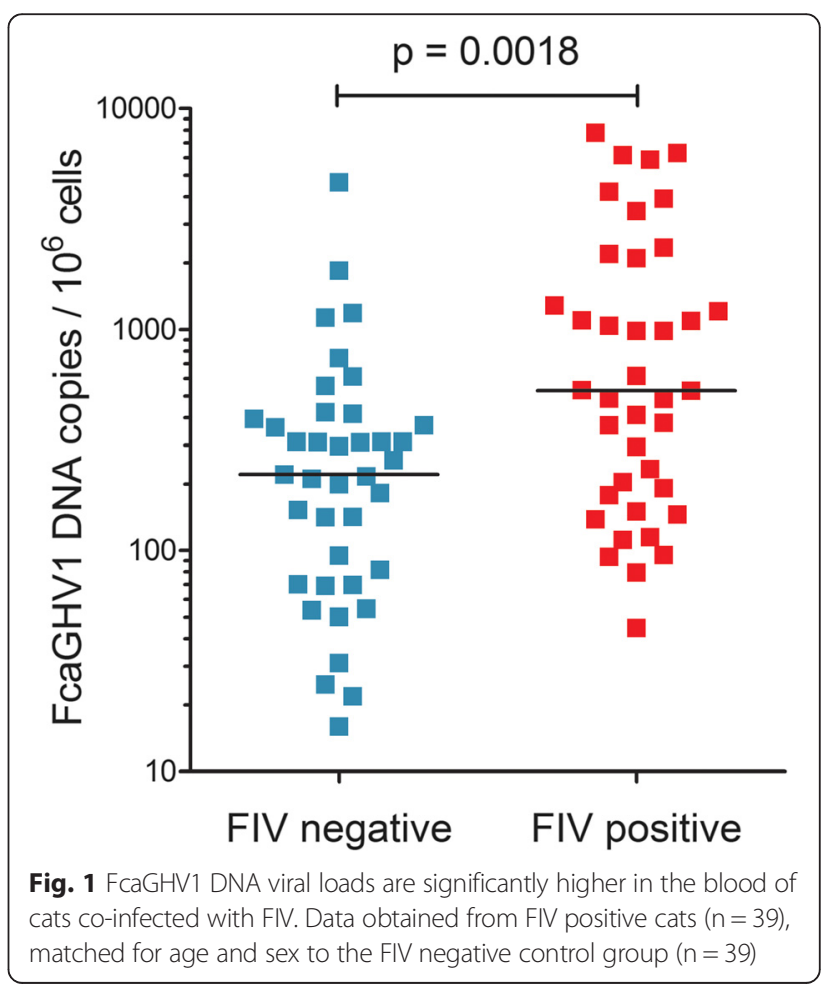


with HIV $[2,8]$. In HIV patients, the virus-induced immunosuppression is presumed to generate an environment that facilitates the oncogenic properties of the GHVs. Thus, virally transformed cells cannot be properly eliminated by the immune system which eventually results in cell proliferation and the development of malignancies [8]. In this context, the increased FcaGHV1 viral loads in co-infected cats might be also a result of the FIV-induced immunosuppression, which possibly enables the reactivation of latent herpesviruses and subsequently enhances the replication of the co-pathogen. Other risk factors that were detected in the present study were related to the cats' demographics. Male and older cats are more likely to be FcaGHV1 positive. These factors have been also described to increase the chance of FIV infection $[9,10]$. No significant differences were detected between intact and neutered cats of the same sex. With regard to FIV, several studies reported a decreased risk of infection for neutered cats, while others found no significant effect of the neuter status [11-13]. When comparing healthy and diseased cats from Austria, we observed slightly greater numbers of virus-positive cats in the diseased group. Though, the statistical associations were not significant. In general, the determination whether a virus infection contributes to an animal's disease status can be challenging in many cases. With the exception of lymphoid malignancies, clinical abnormalities found in FIV-infected cats are generally not significantly different to those of uninfected animals [10]. Additional studies with higher numbers of cats representing better defined disease categories are necessary to further investigate the pathogenic potential of FcaGHV1. Overall, striking similarities for the risk factors of the two viruses can be noted, indicating that both viruses share common transmission routes [4]. FcaGHV1 gB sequence comparisons of 12 German and 6 Austrian isolates with previously reported strains could detect one new nucleotide variation for this gene, found in a single isolate from Austria. As viruses with large dsDNA genomes are generally expected to have rather low mutation rates [14] and especially the FcaGHV1 gB gene is known to be highly conserved [3, 4], longer genome sequences will be needed to get deeper insights into the molecular relationship between different virus isolates.

In summary, our results show that GHV infections are common among European pet cats. Together with previous observations, this indicates that FcaGHV1 is most likely present in cat populations worldwide. FcaGHV1 detection was associated with male sex, increasing age and positive FIV status. Increased viral loads in coinfected animals support the assumption of pathogenic interactions between feline GHVs and FIV.

\section{Methods}

EDTA blood samples were collected from pet cats presented to veterinarians in Germany and Austria in
2012-2015. All samples were taken as a part of the routine clinical examination. DNA was isolated from whole blood using commercial kits. FcaGHV1 qPCR and primers for DNA sequencing of the viral $\mathrm{gB}$ gene have been described [3, 4]. Bi-directional Sanger sequencing was performed by Microsynth, Balgach, Switzerland. The number of cell equivalents for each DNA sample was determined based on qPCR quantification of the feline RPP30 gene [15]. RPP30 copy numbers were used for the calculation of viral loads and as a control for the DNA preparation. The observed host gene numbers in the DNA samples ranged from $1.17 \times 10^{4}-1.72 \times 10^{5}$ copies RPP30/ $2 \mu \mathrm{l}$, which was found to be sufficient to reproducibly detect also low levels of viral DNA. The detection limit of the virus-specific qPCR assay, which was evaluated by a dilution series of FcaGHV1 gB-containing plasmid DNA, was 2 copies per reaction. All cats were tested for FIV provirus DNA by qPCR and conventional PCR as previously described $[16,17]$. Plasmid DNA standards were prepared for all qPCR assays (FcaGHV1, FIV1416p and RPP30) to quantitate the DNA copy numbers. Statistical analyses were performed using the GraphPad Prism 5 software (GraphPad Software, Inc.). A chi-square goodness of fit test and odds ratios were used to compare prevalence rates between the groups. FcaGHV1 viral loads were compared using a two-sided Mann Whitney $U$ test. Spearman's rank correlation coefficient was calculated to assess the relationship between FcaGHV1 and FIV viral loads. Statistical associations were considered significant at $\mathrm{p}<0.05$.

\section{Additional file}

Additional file 1: No correlation of FcaGHV1 and FIV DNA copy

numbers in the blood of co-infected cats. Virus copy and cell numbers were measured by quantitative PCR. Spearman's rank correlation coefficient was calculated to assess the statistical association. (TIFF $10392 \mathrm{~kb}$ )

\section{Abbreviations}

Cl: Confidence interval; EBV: Epstein-Barr virus; FcaGHV1: Felis catus gammaherpesvirus 1; FIV: Feline immunodeficiency virus; gB: glycoprotein B; GHV: Gammaherpesvirus; KSHV: Kaposi's sarcoma-associated herpesvirus; OR: Odds ratio; qPCR: Quantitative PCR.

\section{Competing interest}

The authors declare that they have no competing interests.

\section{Authors' contributions}

Performed experiments and analyzed the data: RE, MK. Wrote the manuscript: RE. Collected sample material and supported data analysis: ILD. Supervised and supported the study: DK. All authors read and approved the final manuscript.

Acknowledgements

We wish to thank Jessica Rushton for critical reading of the manuscript.

\section{Author details}

${ }^{1}$ VetCore Facility for Research, University of Veterinary Medicine Vienna, Vienna, Austria. ${ }^{2}$ Laboklin GmbH \& Co KG, Bad Kissingen, Germany. 
Received: 15 May 2015 Accepted: 10 September 2015

\section{Published online: 17 September 2015}

\section{References}

1. Davison AJ, Eberle R, Ehlers B, Hayward GS, McGeoch DJ, Minson AC, et al. The order Herpesvirales. Arch Virol. 2009;154:171-7.

2. Cesarman E. Gammaherpesvirus and lymphoproliferative disorders in immunocompromised patients. Cancer Lett. 2011;305:163-74.

3. Troyer RM, Beatty JA, Stutzman-Rodriguez KR, Carver S, Lozano CC, Lee JS, et al. Novel gammaherpesviruses in North American domestic cats, bobcats, and pumas: Identification, prevalence, and risk factors. J Virol. 2014;88:3914-24.

4. Beatty JA, Troyer RM, Carver S, Barrs VR, Espinasse F, Conradi O, et al. Felis catus gammaherpesvirus 1 ; a widely endemic potential pathogen of domestic cats. Virology. 2014;460-461:100-7.

5. Hartmann K. Clinical aspects of feline immunodeficiency and feline leukemia virus infection. Vet Immunol Immunopathol. 2011;143:190-201.

6. Magden E, Quackenbush SL, VandeWoude S. FIV associated neoplasms-A mini-review. Vet Immunol Immunopathol. 2011;143:227-34.

7. Beatty J. Viral causes of feline lymphoma: Retroviruses and beyond. Vet J. 2014;201:174-80.

8. Ramos da Silva S, Elgui de Oliveira D. HIV, EBV and KSHV: Viral cooperation in the pathogenesis of human malignancies. Cancer Lett. 2011;305:175-85.

9. Bande F, Arshad SS, Hassan L, Zakaria Z, Sapian NA, Rahman NA, et al. Prevalence and risk factors of feline leukaemia virus and feline immunodeficiency virus in peninsular Malaysia. BMC Vet Res. 2012;8:33.

10. Liem BP, Dhand NK, Pepper AE, Barrs VR, Beatty JA. Clinical Findings and Survival in Cats Naturally Infected with Feline Immunodeficiency Virus. J Vet Intern Med. 2013;27:798-805.

11. Chhetri BK, Berke O, Pearl DL, Bienzle D. Comparison of risk factors for seropositivity to feline immunodeficiency virus and feline leukemia virus among cats: a case-case study. BMC Vet Res. 2015;11:30.

12. Goldkamp CE, Levy JK, Edinboro CH, Lachtara JL. Seroprevalences of feline leukemia virus and feline immunodeficiency virus in cats with abscesses or bite wounds and rate of veterinarian compliance with current guidelines for retrovirus testing. J Am Vet Med Assoc. 2008;232:1152-8.

13. Chang-Fung-Martel J, Gummow B, Burgess G, Fenton E, Squires R. A doorto-door prevalence study of feline immunodeficiency virus in an Australian suburb. J Feline Med Surg. 2013;15:1070-8.

14. Sanjuán R, Nebot MR, Chirico N, Mansky LM, Belshaw R. Viral mutation rates. J Virol. 2010;84:9733-48.

15. Ertl R, Klein D. Transcriptional profiling of the host cell response to feline immunodeficiency virus infection. Virol J. 2014;11:52.

16. Klein D, Leutenegger CM, Bahula C, Gold P, Hofmann-Lehmann R, Salmons B, et al. Influence of preassay and sequence variations on viral load determination by a multiplex read-time reverse transcriptase-polymerase chain reaction for feline immunodeficiency virus. J Acquir Immune Defic Syndr. 2001;26:8-20.

17. Steinrigl A, Klein D. Phylogenetic analysis of feline immunodeficiency virus in Central Europe: A prerequisite for vaccination and molecular diagnostics. J Gen Virol. 2003;84:1301-7.

\section{Submit your next manuscript to BioMed Central and take full advantage of:}

- Convenient online submission

- Thorough peer review

- No space constraints or color figure charges

- Immediate publication on acceptance

- Inclusion in PubMed, CAS, Scopus and Google Scholar

- Research which is freely available for redistribution 